\title{
Back-up circuits: paralogues lend a hand
}

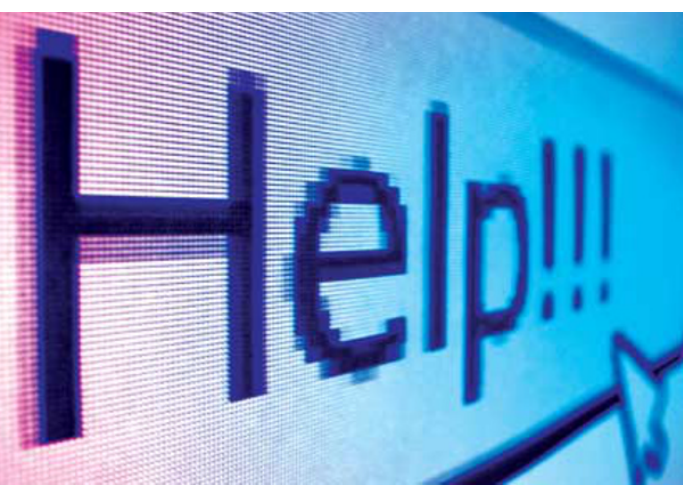

Why do so many severe mutations not show a phenotype? Paralogues are thought to step in and rescue the function of the mutated gene, although new research shows that this backup is - perhaps paradoxically - only seen between paralogues that have dissimilar expression patterns.

Duplicated pairs of genes - paralogues do not exist without a good reason, as genes with identical functions are usually dealt with by forcing one gene copy to diversify or die. A recent study takes its cue from the observation that some diversified gene copies can remember their original function, and can lend support when their paralogues are compromised. By analysing two sets of data for Saccharomyces cerevisiae - mutant viability and mRNA expression - researchers show that this backup is most efficent when the expression pattern of the paralogues is largely non-overlapping, and they have developed a molecular model for how a paralogue fills in for its mutated relative.

It is intuitive that paralogues that are functionally similar are also more likely to substitute for each other in times of need, and it stands to reason that it helps if the two paralogous proteins localize to the same part of the cell: this is indeed the case for S. cerevisiae. The unexpected news, from studying mRNA-expression profiles under 40 growth conditions, was that the best back-up systems involve paralogues with mRNA expression that is only partially correlated. This indicates that, on mutation, the transcription profile of non-mutated paralogues is reprogrammed to match that of the mutated copy. The predictions of this idea were borne out by several experiments; for example, paralogues that show low transcriptional correlation were transcriptionally activated when one copy was mutated. How paralogues might achieve reprogramming was indicated by the architecture of their promoters. Optimal backup occurs when genes feature a mix of regulatory motifs: unique ones, which would govern the differential expression of paralogues in the wild-type, and common ones, which would come into play during back-up.

Perhaps the most captivating questions that arise from this study - How exactly does a mutation trigger the reprogramming of gene expression? Can back-up circuits be selected for? - are still at the modelling stage, but could nevertheless refocus our views of genetic robustness in yeast and beyond.

Tanita Casci

(9) References and links

ORIGINAL RESEARCH PAPER Kafri, R. et al. Transcription control reprogramming in genetic backup systems. Nature Genet. 20 February 2005 (doi:10.1038/ng1523)

FURTHER READING Prince, V. E. \& Pickett, F. B. Splitting pairs: the diverging fates of duplicated genes. Nature Rev. Genet. 3, 827-837 (2002)

WEB SITE

Yitzhak Pilpel's laboratory: http://longitude.weizmann.ac.il 